\title{
Stress-strain State and Vibration Frequencies of Blades of the Main Mine Fan Impeller
}

\author{
N. V. Panova, E. A. Spiridonov \\ N.A. Chinakal Institute of Mining, Siberian Branch of the Russian Academy of Sciences, Novosibirsk, Russia \\ Novosibirsk state technical University
}

Received 2013

\begin{abstract}
The paper discusses geometrical characteristics and strength parameters of fan blades equipped with replaceable active part it end ended to improve the fan adjustability owing to replacement or removal of the active part, which allows the fan to maintain a wide range of ventilation modes.
\end{abstract}

Keywords: Stress-strain State; Vibration Frequencies; Mine Fan Impeller

\section{Introduction}

The main ways to control air purity in a mine are dilution and carryover of mining-induced toxic contaminants using main mine fans.

Main mine fans in existence are uneconomic and accident-sensitive; they take too much space to be located on land surface and, among other shortcomings, are poor-adaptable to mine ventilation mode changes whenever required.

Deficient adjustability results in low technical and economic performance of a ventilation network unit or a main mine fan for most of its running time; or else a main mine fan, after several year of operation, becomes incapable to service this particular ventilation network and, consequently, modification of the ventilation network or construction of new mine fans is required.

To study behavior of ventilation network parameters in terms of actual mines in Russia, Ukraine and Kazakhstan (e.g., Kuznetsk and Donetsk Coal Basins, and others), the representative statistics over a period of 9 to 10 years have been collected and processed. The basic index was chosen the rate of change of the studied parameters, determined as the ratio of the later time-interval value of a parameter to its former time-interval value, the time lengths being varied.

It was found that the rates of change of ventilation parameters in mines are not constant in time, are different in different basins and vary in a wide range; at that, the average capacities are $\mathrm{V}_{\mathrm{Q}}=4.5-15.4 \%$ per year for a fan and $\mathrm{V}_{\mathrm{A}}=-4.0-5.5 \%$ for an effective opening of a ventilation network. Accordingly, if the required fan capacity has been increased by $0.9 \%$ per three months $\left(V_{O}=\right.$ $1.009)$, or by $3.8 \%$ per year, then the fan reserve $(10 \%)$ would be worked out less than in three years, and insufficient performance of the fan would retard development of mining operations.

The analysis of different approaches to enhancement of adaptability of main mine fans [1] showed the possibility to provide the desired ranges for the fan capacity (ten times and higher) and pressure (five times and higher) by using modified axial fans with possible replacement or removal of a number of blades from the fan impeller.

The research into aerodynamics of axial fans, conducted at N.A. Chinakal Institute of Mining, Siberian Branch of the Russian Academy of Sciences [2], showed that it is only possible to enlarge the area of reasonably possible modes of fans in the coordinates Q- $\mathrm{P}$ by designing of axial fans with replaceable, double, sheet blades.

To reach the objectives, fans, series VO, were furnished with replaceable (to be adaptable to different ventilations network standards) and rotable-in-run blades, which allowed continous control over their operation (see Figure 1: within $b$ to $b^{\prime}$; from a to $a^{\prime}$; from $c$ to $c^{\prime}$ ). In this case, the fan adjustment to changes in airing parameters is accomplished by replacement or removal of a number of blades within the modes $\mathrm{a}-\mathrm{a}^{\prime}, \mathrm{b}-\mathrm{b}^{\prime}$ or $\mathrm{c}-\mathrm{c}^{\prime}$, upon variation in air resistance of the ventilation network, within $R_{1}, R_{2}$ and $R_{3}$, respectively (refer to Figure 1 ).

The double, sheet blades designed for axial fans include a rotary base with a root fixing, two special-geometry blades and a connecting strap to maintain the required stiffness of the blades. The double, sheet blades as against profiled blades are advantageous for (1) total elimination of bending and twisting moments of centrifugal forces relative to the fan rotation axis; and (2) 
greatly simplified manufacturing technology.

Figures $\mathbf{2}$ and $\mathbf{3}$ show the calculation schemes for centrifugal forces and moments that influence the double, sheet blades of axial fans.

The double, sheet blades are designed [1] for pre-set parameters of a fan (pressure, capacity, etc.), additionally considering the requirement of zero moment of centrifugal forces on the blades [2], which is achieved by locating center of masses $\mathrm{O}_{1}$ and $\mathrm{O}_{2}$ of the blades so that the center of masses of the entire blade couple is on the axis $\mathrm{Z}$ : moment $\mathrm{M}_{\mathrm{z}}$ is zero (see Figure 2).

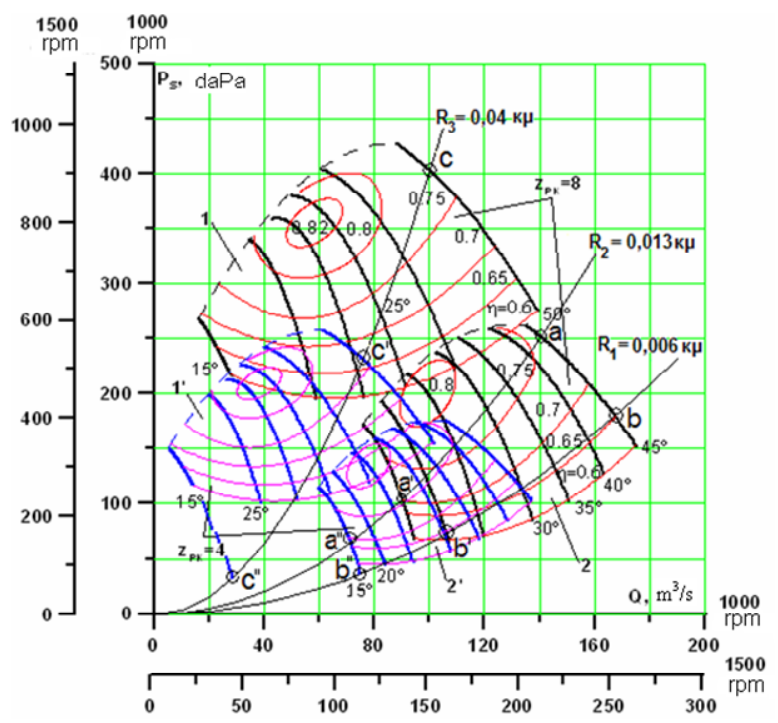

Figure 1. Aerodynamic characteristics of fan, model VO$21 \mathrm{~K}$, at rotation frequency $1000(1500) \mathrm{rpm}$, with blades in amount of $Z_{i m p}=8$ and $Z_{i m p}=4$, mounted on the fan impeller according to aerodynamic schemes AM-17A (1 and 1', high-pressure) and AM-19A (2 and 2', high-capacity), at pitch $\theta=15-50^{\circ}$ and air resistances $R_{1}, R_{2}$ and $R_{3}$.

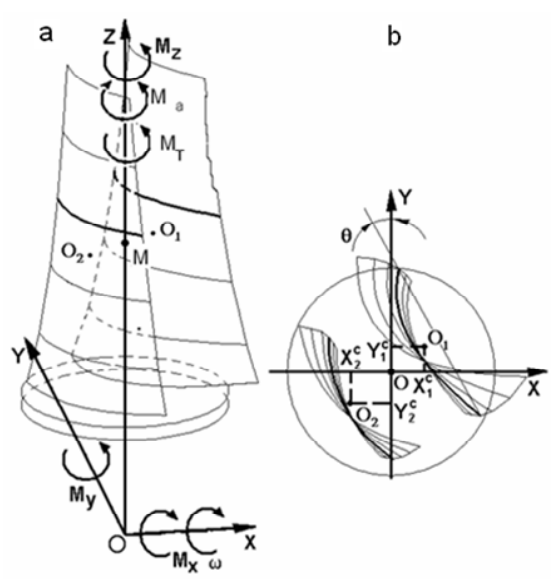

Figure 2. Calculation scheme for moments on fan blades: $M_{x}, M_{y}, M_{z}-$ moments of forces affecting the blades relative to the axes $\mathrm{X}, \mathrm{OY}, \mathrm{OZ}$, respectively; $\omega$-impeller rotation frequency; $\mathrm{O}_{1}$ and $\mathrm{O}_{2}$ - centers of masses of the blades; $\theta$-pitch of the blades.

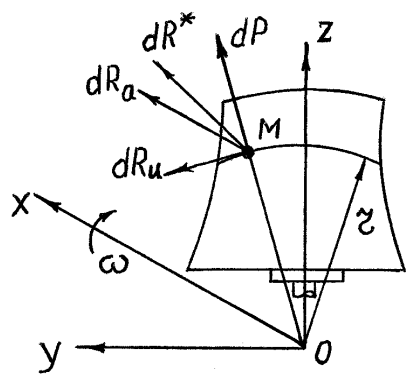

Figure 3. Calculation scheme of forces affecting fan blades.

An illustration of the forces affecting a blade is given in Figure 3, in the coordinates OX, OY, OZ, where it is assumed that the resultant of the forces is at the point $\mathrm{M}$ [2]. It is taken that mine fans rotate counterclockwise if watched from the direction of indraft, and the OX-way projection $\mathrm{dR}^{*}$ is composed of the blade draft force $\mathrm{dR}_{\mathrm{a}}$ and the blade rotational resistance $\mathrm{dR}_{\mathrm{u}}$.

The components $\mathrm{dR}_{\mathrm{a}}$ and $\mathrm{dR}_{\mathrm{u}}$ of the force $\mathrm{dR}^{*}$ are mainly of aerodynamic nature. The OM-way projection $\mathrm{dP}$ of the force $\mathrm{dR}^{*}$ is conditioned by inertia (centrifugal) forces influencing the blades.

The aerodynamic forces $\mathrm{dR}_{\mathrm{u}}$ and $\mathrm{dR}_{\mathrm{a}}$ generate the twisting and bending moments, respectively, while the centrifugal force $\mathrm{dP}$ creates tensile effect and twisting moment on the blades.

Out of the forces $\mathrm{dR}_{\mathrm{u}}, \mathrm{dR}_{\mathrm{a}}$ and $\mathrm{dP}$, the centrifugal force $\mathrm{dP}$ produces the highest load on the holder group of blades, power of impeller and rotation mechanism of blades.

The centrifugal component of mass of blades relative to rotation axis of rotor shaft is calculated from the formula [3]:

$$
P_{z}=m \cdot r \cdot \omega^{2},
$$

where: $m$-is mass of the unit of blades; $r$-distance from center-of-gravity of the unit of blades to the rotation axis; $\omega$-angular rotation frequency of impeller.

The total aerodynamic force is:

$$
F=S_{p r} \cdot P,
$$

where: $S_{p r}=\pi\left(\frac{D}{2}\right)^{2}-\pi\left(\frac{0.6 \cdot D}{2}\right)^{2}$ the blading section area; D-impeller diameter measured between the tips of the blades; $P$-maximal static pressure of fan $(\mathrm{Pa})$.

To make the replacement of impeller blades simpler, for instance, when the blades with other aerodynamic characteristics are required to increase the main mine fan pressure or capacity, the design of the unit of blades has been modified: the base and replaceable part of the unit of blades are fixed using a bolted-type connection (Figure 4).

The internal thread diameter of the bolted-type con- 
nection is selected as follows:

$$
d=\sqrt{\frac{4 N}{\pi \cdot\left[\sigma_{p}\right]}},
$$

where: $N$-external tension force on this particular threaded joint; $\left[\sigma_{p}\right]$-admissible tension stress.

The area pressure exerted by circumference-unequal flow on the surface of a blade, $q(t)$, can be presented as [3]:

$$
q_{(t)}=q_{c o n s}+\Sigma q_{k z} \cdot \operatorname{Sin}\left(2 \pi n k z t+\alpha_{k z}\right),
$$

where: $q_{(t)}$-time-variant load per unit length of blade; $q_{\text {cons - constant load on blade; }}$

$q_{k z}$-amplitude of the time-variant load per unit length of blade at frequency $f=k n z ; 2 \pi n t$-angular coordinate of blade rotation; $\alpha_{K z}-$ phase angle.

Reliability of blades is assured through (1) their sufficient structural strength and (2) absence of resonance phenomena as a response to the nonstationary exposure, e.g., rotor speed, ventilation network vibrations, etc. In the first case it is required to sustain permissible stress in a blade, considering strength limits and load factors of the materials used; the second target is achieved owing to safe tuning-out of the blade vibration frequencies under the excitation forces and, thus, elimination of hazardous dynamic stress under probable resonance effect.

The main way to solving the engineering tasks above is the optimized selection of design variables of blades so that to ensure required strength and frequency. Along with classical approaches, such as material selection and change of cross-sections in strained members of blades, in case of the double, sheet blades, there is an additional option of stress and frequency control by means of numerical modeling of the parameters of connecting straps, stiffening plates, etc.

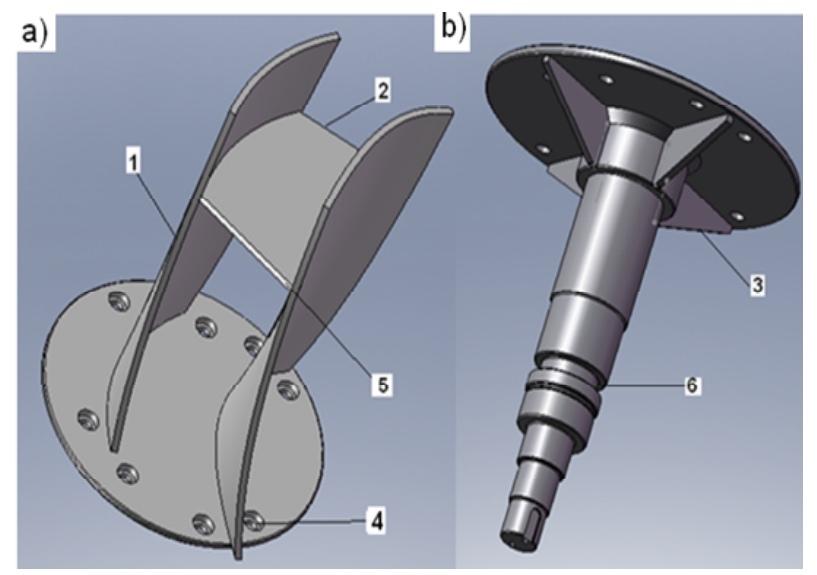

Figure 4. Adaptable unit of blades for fans VO: a-replaceable part; b-rotary base with root fixing; 1 -blades; 2-connecting strap; 3-stiffening plates; 4 -opening for the bolted-type connection; 5-the strap and blade fixture point; 6 - keyseat in the root fixing.
The mentioned above design of a blade unit, but without the replaceable part, was analyzed earlier, though the influence the bolted-type connection has on the strength and resonance of the blade unit was left beyond the analysis. The authors of the present paper have assessed the stress-strain state and frequencies of the adaptable blades, and the stress-strain state of the bolted-type connection between the replaceable part and the base; the principal condition here was the equal strengths of all members of a blade unit and the tuning-out of excitation force at the known frequency.

The strength calculations of different structures commonly use the finite element method that can be successfully accustomed to the developed electronic and computer technologies as well as allows computation of complex objects, including their geometry, load distribution, boundary conditions and physical properties of the materials the analyzed devices are made of.

The analysis of stress-strain state, frequencies and vibrations in the case in question used the ANSYS program, three-dimensional modeling and finite-element modeling. The blades were divided into 3D four-unit finite elements. The bolting modeling involved imposition of zero displacements and rotations; centrifugal forces were calculated automatically, via setting of a special option. As a result, stress-strain state diagrams were obtained for the given design blade unit and a fan with impeller diameters of $2100 \mathrm{~mm}, 2400 \mathrm{~mm}, 3000 \mathrm{~mm}, 3600 \mathrm{~mm}, 4300 \mathrm{~mm}$ and $5000 \mathrm{~mm}$ and rotation frequency range from $375 \mathrm{rpm}$ to $1500 \mathrm{rpm}$.

For every dimension-type of the fans, the authors have determined the following characteristics:

1) Efficient geometrical characteristics and amount of stiffening plates of the rotary base;

2) Permissible thickness of the material sheet to manufacture the blades and connecting straps;

3)O Permissible width of the connecting straps;

4) Stress-strain state and frequencies of the blade unit: individually for the replaceable part and the base;

5) The stress-strain state and frequencies of the blade unit as an assembly.

Table 1 below presents the data of the analysis into the influence of the connecting strap width, given the equal width of the blade sheets, on the stress-strain state and frequency of the blade unit mounted on the fan model VO-43K with impeller diameter of $4300 \mathrm{~mm}$ and speed $\omega=600 \mathrm{rpm}$. The width of the connecting strap was varied as a percentage of the blade chord length in the calculation cross-section; the following strap widthto-chord length percentage ratios were analyzed: $52 \%$, $68 \%, 73 \%, 84 \%, 94 \%$. It has been found that the maximal stresses arise either at the keyseat of the root fixing ( 6 in Figure 4), or at the fixture point of the strap- $2 / 3$ of the blade length from the base ( 5 in Figure 4), depending on the percentage. 
Table 1. Influence of width of the connecting strap on the stress-strain state in the elements of the blade.

\begin{tabular}{ccccc}
\hline $\begin{array}{c}\text { Percentage ratio of the strap width } \\
\text { to the blade chord length, \% }\end{array}$ & $\begin{array}{c}\text { Connecting } \\
\text { strap weight, } \mathrm{kg}\end{array}$ & $\begin{array}{c}\text { Maximal displacements } \\
\text { at the tips of blade, mm }\end{array}$ & $\begin{array}{c}\text { Maximum stresses in the } \\
\text { elements of the blade, MPa }\end{array}$ & $\begin{array}{c}\text { Maximum stress location } \\
\text { in the elements of the blade }\end{array}$ \\
\hline 1 & 2 & 3 & 4 & 5 \\
94 & 9 & 6.3 & 475 & root fixing \\
84 & 8 & 6.2 & 472 & 445 \\
73 & 7 & 6.2 & 466 & root fixing \\
68 & 6.5 & 6.5 & 490 & root fixing \\
52 & 5 & 6.5 & connecting strap \\
connecting strap
\end{tabular}

Table 2. Vibration frequencies of blade units of the VO model fans.

\begin{tabular}{|c|c|c|c|c|c|}
\hline \multirow{2}{*}{ Fan model } & \multicolumn{3}{|c|}{ Rotor speed, tip speed of blades } & \multirow{2}{*}{$\begin{array}{l}\text { Free vibration frequencies, blade } \\
\text { unit }(1 ; 2 ; 3), \mathrm{Hz}\end{array}$} & \multirow{2}{*}{$\begin{array}{l}\text { Factor of safety (resonance tun- } \\
\text { ing-out), } n\end{array}$} \\
\hline & rpm & $\mathrm{Hz}$ & $\mathrm{m} / \mathrm{s}$ & & \\
\hline 1 & 2 & 3 & 4 & 5 & 6 \\
\hline VO-30K & 1000 & 16.7 & 157.3 & $69.97 ; 82.53 ; 198.01$ & $4.2 ; 4.9 ; 11.8$ \\
\hline VO-36K & 750 & 12.5 & 141.3 & $50.63 ; 74.54 ; 155.44$ & $4.0 ; 5.9 ; 12.4$ \\
\hline VO- $43 \mathrm{~K}$ & 600 & 10 & 135.0 & $26.62 ; 89.89 ; 114.58$ & $2.6 ; 8.9 ; 11.4$ \\
\hline VO-50K & 500 & 8.3 & 130.3 & $39.03 ; 79.11 ; 123.84$ & $4.7 ; 9.5 ; 14.9$ \\
\hline
\end{tabular}

It follows from Table 1 that when the connecting strap is reduced in width, the weight of the blade unit gets lower, from $5 \mathrm{~kg}$ to $9 \mathrm{~kg}$, and the centrifugal forces and stresses at the keyseat of the root fixing decrease. After the certain value of the percentage ratio between the strap width and the blade chord length in the calculation cross-section (namely, 68\%), the maximum stresses are observed at the strap fixture point; under further diminution of the connecting strap, the maximum stresses grow and so do the maximum displacements at the tips of the blades. The analysis of the free vibration frequencies of the blade unit has shown that at the percentage ratio between the strap width and the blade chord length of $94 \%$, the first, second and third free frequencies were $26.6 \mathrm{~Hz}$, $89.5 \mathrm{~Hz}$ and $115 \mathrm{~Hz}$; the same for the percentage ratio of $52 \%$ made up $26.3 \mathrm{~Hz}, 91.8 \mathrm{~Hz}$ and $111.7 \mathrm{~Hz}$, respectively. It is seen that the frequencies differ insignificantly, and the required tuning-out of resonance is achieved. The strength assessment included calculation of the factor of safety: $\mathrm{n}=\sigma_{\mathrm{abs}} / \sigma_{\mathrm{appl}}$. For the analyzed blade units of the fans $\mathrm{VO}$, the factor of safety ranges from 1.4 to 4.5.

According to Russian Federation standards for the mine fan designing, the deviation of the blade profile (by tips), vane ring and directing vanes off the nominal profile should be no more than 0.002 of the nominal diameter of fan impeller. Accordingly, allowable deviation by tips of blades for a fan impeller with the diameter of $4300 \mathrm{~mm}$ is $8.6 \mathrm{~mm}$, and the maximum displacements are within the limits (refer to Table 1).

It has been said above that the main reasons for vibra- tions in a blade unit are the free vibration frequency of the fan rotor and the variable aerodynamic flow disturbance in ventilation network. The aerodynamic flow disturbance is conditioned by the unstable resistance in the network. The flow disturbance behavior has sufficiently been studied, and the ample experimental results give evidence that the flow disturbance range lies at the sublow frequencies $\left(f \leq 10^{-3}-10^{-2} \mathrm{~Hz}\right)$. Consequently, the effect of the ventilation network vibrations is low as compared with the influence of the rotor speed (Table 2) and can be withdrawn from calculations.

The accomplished tests have shown that the blade units with the replaceable part meet every demand of strength and durability, namely, such blade units stand permissible stress, displacements and resonance tuningout at the first three vibration frequencies.

\section{Conclusions}

The blade units of fans VO, equipped with replaceable active part possess sufficient safety factor in terms of permissible stress, displacement and resonance tuning-out at the first three vibration frequencies.

Efficient adaptability of axial fans by replacement or removal of active parts of the fan blades, designed according to different aerodynamic schemes, favors wide range of ventilation modes to be maintaned by fans.

\section{Acknowledgements}

Work is executed at financial support of the grant 
№14.B37.21.0333 of July 26, 2012.

\section{REFERENCES}

[1] N. N. Petrov, "Main Axial-Flow Mine Fans with Higher Adaptability, Maintainability and Reliability," Proceedings of the 22nd WMC\&Expo, Istanbul, Turkey, 11-16 September, 2011.
[2] N. N. Petrov, N. A. Popov, E. A. Batyaev and V. A. Novikov, "Theory and Design of Reversible Axial fans with Turn in Motion Blades of Impeller," Journal of Mining Science, Vol.35, No. 5, 1999, pp. 79-97. doi:10.1007/BF02562513

[3] K. N. Borishansky, "Vibration of Turbine Blades," Study Guide, Saint-Petersburg: PIMash, 1995. 